\title{
Chamomile Tea Potentials in Prevention and Amelioration of Type 2 Diabetes Mellitus
}

\section{Mina T Kelleni*}

Department of Pharmacology, Faculty of Medicine, Minia University, Egypt

\section{Commentary}

A recent randomized controlled trial has confirmed many previous animal studies suggesting chamomile tea to contribute to the prevention of the progress of hyperglycemia and diabetic complications. In this commentary, a concise review of chamomile tea and its relationship with type 2 diabetes mellitus is provided.

Matricaria (Chamomilla) recutita L, known as "chamomile", has been used as an herbal tea or supplementary food all over the world. It has a long history of being used as a medicinal plant due to many health benefits, including anti-inflammatory, anticancer, antispasmodic, radical-scavenging effects and others [1]. Moreover, chamomile extract was shown to have a potent antidiarrheal and antioxidant properties in rats confirming their use in traditional medicine [2]. In 2008, Khan and colleagues indicated that chamomile tea possesses a glucose lowering effect in diabetic rats, other researchers confirmed the ability of chamomile to progressively reduce the fasting and post prandial blood sugar levels as well as lowering the level of hemoglobin Alc (HbA1c) significantly suggesting a possible potential in type 2 diabetes management and recommending its daily consumption as a potentially useful tool in hyperglycemia control $[3,4]$. One of the documented antioxidants isolated from chamomile tea is luteolin; a flavone found also in celery, green pepper, perilla leaf and has been reported to possess antimutagenic, antitumorigenic, antioxidant, and anti-inflammatory properties [4,5]. Other documented chamomile antioxidant extracts include apigenin, umbelliferone, esculetin and quercetin. Some of these extracts were shown to inhibit the accumulation of sorbitol in human erythrocytes as well as to significantly suppress the blood glucose levels in streptozotocin-induced rat diabetes model and it was suggested that daily consumption of chamomile tea with meals could contribute to the prevention of the progress of hyperglycemia and diabetic complications [3].

Further, Fitzenberger and colleagues have shown that extracts made from chamomile, ginseng, lime blossom, paraguay tea, balm, rhodiola, black tea, or knotgrass all significantly extended the lifespan and ameliorated the glucose-induced reduction of survival in nematodes [6]. Further, researchers from Tabriz University of medical sciences have shown that type 2 diabetes mellitus patients consuming chamomile tea three times per day immediately after meals for eight weeks has beneficial effects on glycemic control significantly reducing $\mathrm{HbA} 1 \mathrm{c}$ as well as significantly increasing total antioxidant capacity compared to a group of patients drinking water only and concluded that chamomile tea could be useful in reducing diabetes risk factors [7]. Interestingly, the same group of researchers added in another publication of the same research that consuming chamomile tea can significantly improve serum lipid profile in T2DM patients [8]

I believe that waiting seven years was too long to confirm the preliminary positive data regarding the antidiabetic effects of chamomile tea in rats published in 2008 with a randomized controlled trial and I do agree with all the researchers who asked for more clinical trials including larger numbers of volunteers to explore more about the protective and/or ameliorative effect of this widely used cheap herbal extract wishing to announce a global recommendation to use it as a daily routine healthy habit before the lapse of seven more years.

\section{References}

1. Cvetanovic A, Svarc-Gajic J, Zekovic Z, Savic S, Vulic J, et al. (2015) Comparative analysis of antioxidant, antimicrobiological and cytotoxic activities of native and fermented chamomile ligulate flower extracts. Planta 242: 721 732

2. Sebai H, Jabri MA, Souli A, Rtibi K, Selmi S, et al. (2014) Antidiarrheal and antioxidant activities of chamomile (Matricaria recutita L.) decoction extract in rats. Journal of ethnopharmacology 152: 327-332.

3. Kato A, Minoshima Y, Yamamoto J, Adachi I, Watson AA, et al. (2008) Protective effects of dietary chamomile tea on diabetic complications. Journal of agricultural and food chemistry 56: 8206-8211.

4. Khan SS, Najam R, Anser H, Riaz B, Alam N (2014) Chamomile tea: herbal hypoglycemic alternative for conventional medicine. Pakistan journal of pharmaceutical sciences 27: 1509-1514

5. Kim HJ, Lee W, Yun JM (2014) Luteolin inhibits hyperglycemia-induced proinflammatory cytokine production and its epigenetic mechanism in human monocytes. Phytotherapy research 28: 1383-1391.

6. Fitzenberger E, Deusing DJ, Wittkop A, Kler A, Kriesl E, et al. (2014) Effects of plant extracts on the reversal of glucose-induced impairment of stressresistance in Caenorhabditis elegans. Plant foods for human nutrition 69: 7884.

7. Zemestani M, Rafraf M, Asghari-Jafarabadi M (2015) Chamomile tea improves glycemic indices and antioxidants status in patients with type 2 diabetes mellitus. Nutrition [Epub ahead of print]

8. Rafraf M, Zemestani M, Asghari-Jafarabadi M (2015) Effectiveness of chamomile tea on glycemic control and serum lipid profile in patients with type 2 diabetes. Journal of endocrinological investigation 38: 163-170.

*Corresponding author: Mina T Kelleni, Department of Pharmacology, Faculty of Medicine Minia University, Egypt, Tel: +201200382422; E-mail: drthabetpharm@yahoo.com

Received November 02, 2015; Accepted February 17, 2016; Published February 23, 2016

Citation: Kelleni MT (2016) Chamomile Tea Potentials in Prevention and Amelioration of Type 2 Diabetes Mellitus. J Diabetes Metab 7: 649. doi:10.4172/2155-6156.1000649

Copyright: ( 2016 Kelleni MT. This is an open-access article distributed under the terms of the Creative Commons Attribution License, which permits unrestricted use, distribution, and reproduction in any medium, provided the original author and source are credited. 